\title{
Optimal Pollution, Optimal Population, and Sustainability
}

\author{
Ulla Lehmijoki \\ University of Helsinki and HECER
}

Discussion Paper No.101

March 2006

ISSN 1795-0562

HECER - Helsinki Center of Economic Research, P.O. Box 17 (Arkadiankatu 7), FI-00014 University of Helsinki, FINLAND, Tel +358-9-191-28780, Fax +358-9-191-28781,

E-mail info-hecer@helsinki.fi, Internet www.hecer.fi 


\title{
Optimal Pollution, Optimal Population, and Sustainability*
}

\begin{abstract}
This paper develops a long-run consumer optimization model with endogenous pollution and endogenous population. The positive check increases mortality if pollution increases. The optimal path is sustainable if it provides non-decreasing consumption for a nondecreasing population. As usually, optimality and sustainability may conflict; with population endogenous to pollution, this conflict may ultimately lead mankind toward selfimposed extinction. Not even technical progress can warrant sustainability. The warning signal is the increasing per capita consumption.
\end{abstract}

JEL Classification: Q01, Q53, Q54, J11

Keywords: sustainable development, mortality, optimal pollution.

Ulla Lehmijoki

Department of Economics,

University of Helsinki

P.O. Box 17 (Arkadiankatu 7

FI-00014 Helsinki

FINLAND

e-mail: ulla.lehmijoki@helsinki.fi

* I would like to thank Pertti Haaparanta and Adriaan Perrels for their constructive comments and The Yrjö Jahnsson Foundation for generous financial support. 


\section{Introduction}

Is it possible that current utility maximization takes place at the cost of human lives? Is it even possible that this maximization will ultimately lead mankind toward self-imposed extinction? These possibilities are implied in the longrun consumer optimization models of Dasgupta and Heal (1974), Solow (1974), Stiglitz (1974), Krautkraemer (1985), and Pezzey and Withagen (1998). They argue that the scarcity of natural resources may lead to ever-decreasing per capita consumption. Per capita consumption may also decrease if excessive pollution impairs production and compromises life-supporting systems as is argued in Keeler et al. (1971), Plourde (1972), Foster (1973) and Smulders and Gradus (1996). In all these models population keeps constant or grows at an exogenously given rate even if per capita consumption decreases.

In this paper, I assume explicitly that population is endogenous to environment. There is a feedback to mortality if population is not environmentally supported; this feedback defined as a "positive check" by Robert Malthus (Malthus 1914). The positive check may occur because of increasing scarcity of resources or because of continuing concentration of pollutants. I focus on pollutants where emerging evidence maintains that the positive check is already at work. This evidence consists of medical and econometric studies performed by individual researchers and international organizations whose main argument is that there is a statistically significant increase in mortality due to urban air pollution, and that climate change may induce further increases in the immediate future. Other global concerns, such as pollution of ground waters and oceans, 
are also possible, but less evidence on their mortality effects has accumulated thus far. In spite of my emphasis on pollutants, the results can be generalized to include natural resources because running down resources can be seen as pollution in an extended sense (Keeler et al. 1971). Thus I provide a model of optimal pollution with population endogenous to pollution. Therefore, by the choice of optimal pollution, optimal population also becomes determined.

Since times, people have been questing for an apocalyptic sign. In earlier studies, several authors show that, along the optimal path, the per capita consumption typically first increases and then decreases (Dasgupta and Heal 1974, Pezzey and Withagen 1998). As the per capita consumption currently everywhere increases, those in search of a sign easy off. But if population is endogenous, and if it is optimal to let population to decrease forever, then along the optimal path per capita consumption first decreases and then increases. Thus, increasing per capita consumption signals that environmental constraint is getting binding. The importance of the correct signal is in the disparity between the model and real life. In the model, there is perfect foresight but in real life the long-run consequences of our current choices are, unfortunately, less transparent.

Section 2 of this paper reviews the empirical evidence on the positive check. Sections 3 and 4 introduce the model and its sustainability implications together with a parametric example; a new definition for sustainability is provided. To concentrate on population, only the simplest model is presented. Even so, endogenous population tends to make the model "murky" (Solow 1974) but exces- 
sive complexity can be avoided by modelling in virtual time. Section 5 discusses the role of technical progress, which in this model is not as positive as usually suggested. Section 6 closes the paper.

\section{The Positive Check - Recent Evidence}

Mortality induced by air pollution has been debated since the smog in the Meuse Valley in 1930 and London in 1952 took the lives of 60 and 4000 people respectively (Nemery et al. 2001 and Logan 1953). Air pollution consists of several components, of which particulate matter $(P M)$ and ozone are the most dangerous (WHO 2004b). ${ }^{1}$ Air pollution increases mortality mainly through an increase in respiratory and cardiovascular diseases and lung cancer (Samet et al. 2000), but an increase in skin cancer is also reported (Brunekreef and Holgate 2002). All age groups are affected, but unborn and young children as well as the elderly are the most vulnerable.

The Clean Air for Europe program $(C A F E)$ and $W H O$ have summarized the European research by collecting 629 peer-reviewed time-series studies and 160 individual or panel studies up to February 2003 (WHO 2004b). In the original studies, daily adult mortality in several European cities was regressed against daily changes in air pollution. The summary estimates indicate that there is a statistically significant $0.6 \%$ and $0.3 \%$ increase in mortality for each

\footnotetext{
${ }^{1}$ The term particulate matter $(P M)$ refers to solid airborne particles of varying size, chemical composition and origin. For example, the particles in $P M_{10}$ have a diameter of less than $10 \mathrm{em}$ and are mainly combustion-derived, either from traffic or from energy production, often from long-distance sources. There is evidence to suggest that the smaller the particles are, the more deeply into the lung they penetrate (WHO 2004b).
} 
$10 \mu \mathrm{g} / \mathrm{m}^{3}$ increase in $P M$ and ozone respectively. ${ }^{2}$

The effects of long-term $P M$ exposure in the United States has been analyzed by Pope et al. (2002) in a study in which a questionnaire from 1982 provides data on sex, race, smoking, alcohol consumption, etc., so that controlling for alternative risk sources has been possible. The mortality data were collected until 1998 and were regressed against local pollution data to derive 4\%,6\%, and $8 \%$ increases in all-cause, respiratory, and lung cancer mortality respectively for each $10 \mu \mathrm{g} / \mathrm{m}^{3}$ increase in $P M .^{3}$ Evans and Smith have recently estimated similar increases (Evans and Smith 2005). The estimates of Pope et al. (2002) have been applied to the European data by $C A F E$ and $W H O$ to calculate that the short-term and long-term exposures were together responsible for 370000 premature deaths in 2000 in Europe (WHO 2004b).

The Clean Air for Europe program and WHO have also provided a synthesis on air pollution and child mortality (WHO 2004a), based on several original studies. The conclusion is given on a four-level scale from "sufficient" to "no association." It turns out that there is sufficient evidence from the increase in child mortality, mainly due to exposure to particulate matter, but no exact summary estimate is provided. The infant mortality risk in California during the $1990 s$ has been estimated by Currie and Neidell (2004), who applied several covariates and controlled for fetal deaths to exclude the selection bias. The pollutants were particulate matter, ozone, carbon monoxide, and nitrogen dioxide.

\footnotetext{
${ }^{2}$ This type of meta-analysis tends to have a "publication bias" because the publication of positive results is more likely in the original studies. The authors tried to correct the bias with the outcome that the risk estimate for ozone decreased to $0.02 \%$ (WHO 2004b).

${ }^{3}$ The Cox proportional hazards survival model was used.
} 
Single pollutant models supplied significant estimates, but when all four were included, only carbon monoxide was significant. Chay and Greenstone (2003) have also shown that the air quality improvement under the Clean Air Act of 1970 in the United States saved more than 1,300 infants annually.

In climate-change studies, the mortality estimates are based on simulations. Tanser et al. (2003), for example, have applied the Hadley Centre's climate model to estimate that the increase in malaria distribution and the prolonged malaria season would lead to a $25 \%$ increase in risk of death from malaria by 2100, mainly in Africa. The abundant literature on climate change has been collected and analyzed by the UN's Intergovernmental Panel on Climate Change (IPCC). Its Third Assessment Report suggests that mortality will increase because of weather extremes, environmental changes that lead to diseases and water or food shortages, and health consequences or conflicts in displaced populations (IPCC 2001). Relying on the IPCC, WHO has published a summary report on human health and climate change (WHO 2003). This report projects a maximum increase in the risk of $83 \%, 17 \%$, and $32 \%$ for the great killers; malaria, diarrhoea, and malnutrition, respectively. There is also a great projected risk increase in coastal floods, but the number of deaths may be low at the global level. 


\section{The Model}

To model the positive check, note that the population growth rate $\dot{L} / L=n$ is the difference between fertility and mortality. In what follows, I assume that mortality depends on pollution but fertility is constant. ${ }^{4}$ Pollution may increase mortality (decrease population growth) both as emissions $E$ and as stocks $S$, but it seems appropriate to model in terms of stocks because their mortality effects are more longstanding. Hence, let:

$$
n=n(S), n(0)>0, n^{\prime}(S)<0, n(\hat{S})=0
$$

where $\hat{S}$ is the critical stock beyond which population starts to decrease. Normalizing the initial level to unity it holds

$$
L(t)=\exp \int_{0}^{t} n[S(\tau)] d \tau
$$

The pollution stock accumulates according to

$$
\dot{S}=E-\delta(S)
$$

where $\delta(S)$ is the abatement function. Let $\delta(0)=\delta(\tilde{S})=0$ and $\delta^{\prime}(0)>$ $0, \delta^{\prime}(\tilde{S})<0, \delta^{\prime \prime}(S)<0$ where $\tilde{S}>0$ is the carrying capacity of the environment. ${ }^{5}$ The abatement function then has the shape of inverted $U$. I assume

\footnotetext{
${ }^{4}$ The United Nations has estimated that the currently ongoing fertility transition will largely be over about 2050 (United Nations 2005).

${ }^{5}$ The abatement function here is strictly concave. A broad branch of literature deals with
} 
$\tilde{S}>\hat{S}$, to allow the possibility of negative population growth in the area of interest. By writing $E=(E / L) \cdot L$, one sees that the environmental burden of population comes from two sources, namely from an increase in per capita emission $E / L$ and from an increase in the number of people $L$.

Consider an infinitely living central planner who wants to maximize societal welfare or total utility. ${ }^{6}$ Let the function for total utility be of multiplicative form. At each instant of time, the total utility then becomes $u(C / L) \cdot L$, where $u$ satisfies the standard concavity properties and the Inada conditions. In her intertemporal choice, the planner faces the discount factor $\rho>0$. To focus on population and pollution in the absence of production problems, I assume the simplest formulation for the rest of the model in line with Foster (1973). Thus, consumption $C$ takes place directly at the cost of environment so that $C=E$. The planner then chooses emissions $E(t)$ to maximize

$$
\begin{aligned}
U & =\int_{0}^{\infty} u[E(t) / L(t)] L(t) e^{-\rho t} d t \\
& =\int_{0}^{\infty} u[E(t) / L(t)] e^{-\int_{0}^{t}\{\rho-n[S(\tau)]\} d \tau} d t,
\end{aligned}
$$

subject to (3.3). The mechanism of the model is the following: by choosing the optimal path for $E(t)$, the planner determines $S(t)$, which in turn gives the optimal population growth rate $n(t)$ and the optimal population $L(t)$. Finally,

the problem of non-concavities in the abatement function; for a survey, see Tahvonen and Salo (1996).

${ }^{6}$ The model pays no attention to amenity values of environment considered by Krutilla (1967) and Barbier (2003). 
per capita emissions $E(t) / L(t)$ get determined.

Because the discount factor in (3.4) is not constant, we apply the virtual time technique suggested by Uzawa (1968). Let us denote

$$
\Delta(t)=\int_{0}^{t}\{\rho-n[S(\tau)]\} d \tau
$$

to get $\frac{d \Delta(t)}{d t}=\rho-n[S(t)]$ and $d t=\frac{d \Delta(t)}{\rho-n[S(t)]}$. The problem can now be rewritten in virtual time as:

$$
\begin{gathered}
U=\int_{0}^{\infty} \frac{u(E / L)}{\rho-n(S)} \cdot e^{-\Delta} \cdot d \Delta, \\
\stackrel{S}{S}=\frac{d S}{d \Delta}=\frac{d S}{d t} \frac{d t}{d \Delta}=\frac{E-\delta(S)}{\rho-n(S)},
\end{gathered}
$$

where $E=E[\Delta(t)], S=S[\Delta(t)], L=L[\Delta(t)]$. This concave problem with constant discount factor can be solved in virtual time by using standard methods (Benveniste and Scheinkman 1982). The current value Hamiltonian and the necessary conditions become:

$$
\begin{gathered}
H(S, E, \lambda)=\frac{1}{\rho-n(S)}\{u(E / L)+\lambda(\Delta)[E-\delta(S)]\}, \\
\frac{\partial H(S, E, \lambda)}{\partial E}=0 \Longleftrightarrow-u^{\prime}(E / L)=\lambda(\Delta) \cdot L, \\
\dot{\lambda}=\frac{d \lambda(\Delta)}{d \Delta}=-\frac{\partial H}{\partial S}+\lambda(\Delta), \\
\lim _{\Delta \rightarrow \infty} \lambda(\Delta) e^{-\Delta} S=0 .
\end{gathered}
$$


Taking the derivative in (3.6) and rearranging we get

$$
\grave{\lambda} / \lambda=-(1 / \rho-n)\left\{n^{\prime} H / \lambda-\left(\delta^{\prime}+\rho-n\right)\right\} .
$$

To eliminate $\lambda$, we follow the usual procedure by taking the derivative of (3.5) in terms of time. In this case, virtual time is relevant. The derivatives are denoted by $\stackrel{\circ}{E}=d E / d \Delta$ and $\stackrel{\circ}{L}=d L / d T$. To simplify the analysis, we adopt the CIES utility function $u(E / L)=\left[(E / L)^{1-\theta}\right] /(1-\theta), \theta /=1$ with $u^{\prime \prime}$. $(E / L) / u^{\prime}=-\theta$. We get

$$
\stackrel{\circ}{\lambda} \lambda=-\theta \stackrel{\circ}{E} / E+(\theta-1) \stackrel{\circ}{L} / L,
$$

which together with (3.8) gives $\stackrel{\circ}{E} / E=[1 / \theta(\rho-n)]\left\{-n^{\prime} H / \lambda-\left(\delta^{\prime}+\rho-\theta n\right)\right\}$, where $\stackrel{\circ}{L} / L=n /(\rho-n)$ is applied. Substituting the expression $-n^{\prime} H / \lambda=$ $\left[n^{\prime} /(\rho-n)\right][\theta E /(\theta-1)-\delta]$ and noting $\stackrel{\circ}{E}=\dot{E} /(\rho-n)$ we finally derive

$$
\frac{\dot{E}}{E}=\frac{1}{\theta}\left\{\frac{n^{\prime}}{\rho-n}\left[\frac{\theta E}{\theta-1}-\delta\right]-\left(\delta^{\prime}+\rho-\theta n\right)\right\}
$$

The non-linear equations (3.3) and (3.10) supply the solution to the model. The phase lines become:

$$
\begin{aligned}
\frac{\dot{E}}{E} & =0 \Leftrightarrow E=\frac{\theta-1}{\theta}\left\{\delta+\frac{\rho-n}{n^{\prime}}\left(\delta^{\prime}+\rho-\theta n\right)\right\}, \\
\dot{S} & =0 \Leftrightarrow E=\delta .
\end{aligned}
$$


In the $(S, E)$-space, the shape of the $\dot{S}=0$-line is that of $\delta$, i.e., inverted $U$ with $\delta(0)=\delta(\tilde{S})=0$. The shape of the $\dot{E}=0$-line depends on the value of $\theta$. Because Hall has argued that empirical elasticities tend to be large (Hall 1988), we assume $\theta>1$, but nothing essential is changed if $\theta<1$ is assumed. Even so, there is variety in the shape of the $\dot{E}=0$-line. The following is the sufficient condition for the existence of at least one interior steady state:

Lemma 1 If $\delta^{\prime}(0)+\theta n(0)>\rho$ and $\delta^{\prime}(\tilde{S})+\theta n(\tilde{S})<\rho$ then the problem has a steady state $S^{*} \subset(0, \tilde{S})$.

Proof. In the $(S, E)$-space the $\dot{S}=0$-line hits the $S$-axis at $S=0$ and at $S=\tilde{S}$. For $S=0$ and $S=\tilde{S}$, (3.11a) then becomes $\dot{E}=0 \Leftrightarrow E=$ $\frac{\theta-1}{\theta}\left\{\frac{\rho-n}{n^{\prime}}\left(\delta^{\prime}+\rho-\theta n\right)\right\}$. By assumption, $\theta-1>0, \rho-n>0$ and $n^{\prime}<0$. Graphically, if $\delta^{\prime}(0)+\theta n(0)>\rho$ and $\delta^{\prime}(\tilde{S})+\theta n(\tilde{S})<\rho$, the $\dot{E}=0$-line lies below the $\dot{S}=0$-line for $S=0$ and above it for $S=\tilde{S}$ (Figure 3.1). By continuity, the $\dot{E}=0$-line intersects the $\dot{S}=0$-line at least once.

To comprehend, consider a marginal emission $E$. If consumed tomorrow, it is discounted by $\rho$. If consumed today, it adds to the pollution stock $S$ and produces a change in abatement $\delta^{\prime}(S)$ and population $n(S)$. If the sum of the latter two is larger, consumption today pays. The first unit of emission is consumed if $\delta^{\prime}(0)+\theta n(0)>\rho$. On the other hand, if $\delta^{\prime}(\tilde{S})+\theta n(\tilde{S})<\rho$ it never pays to pollute until the carrying capacity $\tilde{S}$.

In what follows, we assume that the number of steady states is one. Standard local analysis shows that this steady state is a saddle with stable manifolds running from the north-west and South-East (see Appendix A), as Figure 3.1 


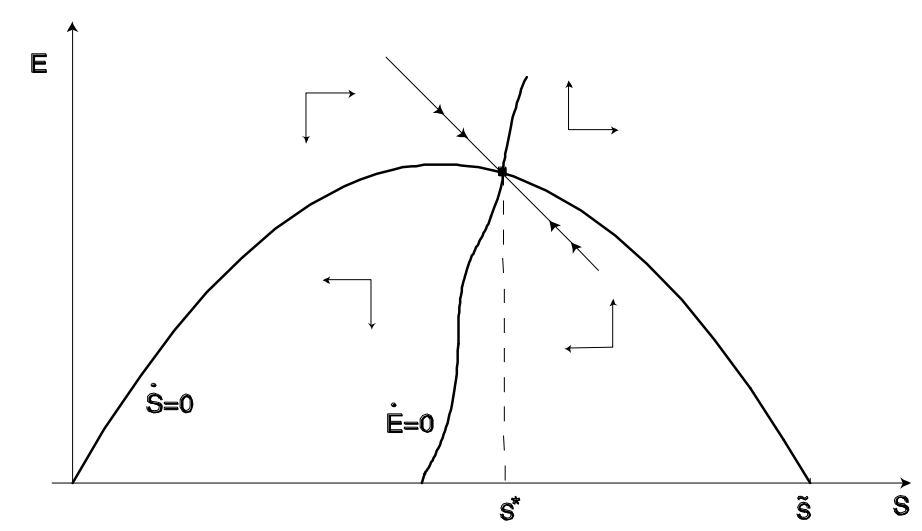

Figure 3.1: The phase diagram of the model.

illustrates. The following lemma characterizes this steady state:

Lemma 2 Inefficient under-accumulation of the pollutant is not possible.

P r oof. Equations (3.11a) and (3.11b) imply that in a steady state

$$
\frac{\theta-1}{\theta}\left\{\delta+\frac{\rho-n}{n^{\prime}}\left[\delta^{\prime}+\rho-\theta n\right]\right\}=\delta .
$$

The transversality condition is $\lim _{\Delta \rightarrow \infty}\left\{\lambda(\Delta) e^{-\Delta} S(\Delta)\right\}=0$. Because the model tends to the steady state, $S$ and $n(S)$ go to constants $S^{*}$ and $n\left(S^{*}\right)$. In a steady state, $\stackrel{\circ}{E}=0$ so that (3.9) implies $\dot{\lambda} / \lambda=(\theta-1) \stackrel{\circ}{L} / L$, which is a constant in the steady state. The transversality condition then requires $(\theta-1) \stackrel{\circ}{L} / L-1<0$. Because $\stackrel{\circ}{L} / L=n /(\rho-n)$, we get $(\theta-1) n\left(S^{*}\right) /\left(\rho-n\left(S^{*}\right)\right)-1<0$ and further

$$
\rho-\theta n\left(S^{*}\right)>0
$$


Arranging and using (3.12) we get $\rho-\theta n=\frac{n^{\prime}}{(\rho-n)(\theta-1)} \delta-\delta^{\prime}>0$. Because $\frac{n^{\prime}}{(\rho-n)(\theta-1)} \delta<0$, it must be $\delta^{\prime}\left(S^{*}\right)<0$. Therefore, the steady state is located on the downwards sloping part of the $\dot{S}=0$-line.

\section{Sustainability}

The Brundtland Comission 1987 defines sustainable development as a development that "meets the needs of the present without compromising the ability of future generations to meet their own needs" (WCED 1987). This definition refers to non-decreasing consumption or non-decreasing utility, concepts also used by most economists (for a review, see Pezzey 1992). With the positive check present, the concept of sustainability needs a redefinition:

Definition 1 An optimal path is sustainable if it provides non-decreasing consumption for a non-decreasing population.

Thus, an optimal path can lose sustainability either because per capita consumption decreases or because the population decreases.

Consider first the steady state. Recall that $E=C$. The growth rate of the per capita consumption is $\gamma_{C / L}=\dot{E} / E-\dot{L} / L$. In the steady state, $E$ is constant so that $\gamma_{C / L}=-\dot{L} / L=-n\left(S^{*}\right)$. Three alternatives are possible. For $n\left(S^{*}\right)>0$, the population keeps increasing and per capita consumption decreasing. For $n\left(S^{*}\right)=0$, both the population and per capita consumption are constants. For $n\left(S^{*}\right)<0$, an ever-decreasing population enjoys ever-increasing per capita consumption. Note that this steady state implies $\lim _{t \rightarrow \infty} L(t)=0$ so 
that, asymptotically, the population becomes extinct. Of the above alternatives, only $n\left(S^{*}\right)=0$ is sustainable.

To stipulate per capita consumption $\gamma_{C / L}=\dot{E} / E-n$ during the off-steadystate transitional period, assume that the economy starts with zero initial pollution stock and then moves towards the steady state along the north-western saddle path (Figure 3.1). Equation (3.10) can be rewritten as

$$
\begin{aligned}
\frac{\dot{E}}{E} & =\frac{1}{\theta}\left\{\frac{n^{\prime}}{\rho-n}\left[\frac{\theta E-\theta \delta}{\theta-1}\right]+\frac{n^{\prime}}{\rho-n} \frac{1}{\theta-1} \delta-\left[\delta^{\prime}+(\rho-\theta n)\right]\right\} \\
& =\frac{n^{\prime}}{(\rho-n)(\theta-1)}\left\{\dot{S}+\frac{1}{\theta}\left[\delta-\frac{(\rho-n)(\theta-1)}{n^{\prime}}\left[\delta^{\prime}+(\rho-\theta n)\right]\right],\right.
\end{aligned}
$$

where $(1 / \theta)\left\{\delta-\left[(\rho-n)(\theta-1) / n^{\prime}\right]\left[\delta^{\prime}+(\rho-\theta n)\right]\right\}$ is the difference between the $\dot{S}=0$ and $\dot{E}=0$-lines which is positive for $S \subset\left(0, S^{*}\right)$ (Figure 3.1). Because $\dot{S}>0$ along the north-western branch and $n^{\prime}(S)<0$ by assumption, we have $\frac{\dot{E}}{E}<0$ for all $S \subset\left(0, S^{*}\right)$.

Now consider $\gamma_{C / L}=\dot{E} / E-n$. By assumption, $n(0)>0$ so that $\gamma_{C / L}<0$ for $S=0$. Because $\lim _{S \rightarrow S^{*}} \dot{E} / E=0$ we have $\lim _{S \rightarrow S^{*}} \gamma_{C / L}=-n\left(S^{*}\right)$. The above three cases again appear. If $n\left(S^{*}\right)>0$, we have $\gamma_{C / L}<0$ for all $S \subset\left(0, S^{*}\right)$ and $\lim _{S \rightarrow S^{*}} \gamma_{C / L}<0$. If $n\left(S^{*}\right)=0$, we have $\gamma_{C / L}<0$ for all $S \subset\left(0, S^{*}\right)$ and $\lim _{S \rightarrow S^{*}} \gamma_{C / L}=0$. Because $\dot{S}>0$ along the north-western branch, these two cases imply that the time path for per capita consumption $E / L$ is decreasing and approaches the steady-state limit $E^{*} / L^{*}$, which is zero 
for $n\left(S^{*}\right)>0$ and positive for $n\left(S^{*}\right)=0 .{ }^{7}$ Finally, if $n\left(S^{*}\right)<0$ we have $\lim _{S \rightarrow S^{*}} \gamma_{C / L}>0$. Because $\gamma_{C / L}<0$ for $S=0$, there exists at least one $S$ such that $\gamma_{C / L}=0$ by continuity of $\gamma_{C / L}$. Therefore, the implied $E / L=C / L$ first decreases and then increases as is shown in Figure 4.1.

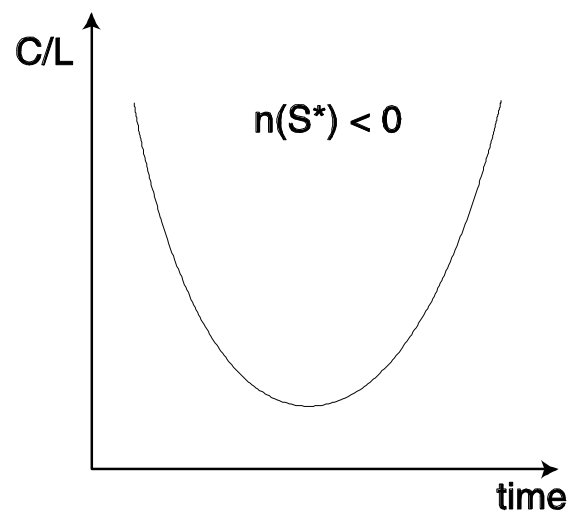

Figure 4.1: The time paths for per capita, the case $n\left(S^{*}\right)<0$.

Which of the above cases realizes? First note that the a priori assumptions $\rho>0$ and $\rho-n(S)>0$ pose no explicit limit to $\operatorname{sign} n\left(S^{*}\right)$. Another candidate that would limit sign $n\left(S^{*}\right)$ is the transversality condition in (3.13) but for suitable values of $\rho$ and $\theta$ it can hold for positive and negative values of $n\left(S^{*}\right)$. Therefore, in the steady state $S^{*}$ the optimal population may be constant, increasing, or decreasing because the utilitarian objective functional $\int_{0}^{\infty} u(E / L) L e^{-\rho t} d t$ may take its maximum both at high $E / L$ and low $L$ or vice versa. Therefore, it may well be optimal to choose increasing consumption at the cost of population.

\footnotetext{
${ }^{7}$ This implies that, from the transitional point on view, the alternative $n\left(S^{*}\right)=0$ is not sustainable, either.
} 


\subsection{A Parametric Example}

Chapter 2 reports some recent evidence of the positive check, but there is only slight evidence on the functional formula by which this check cuts in. However, some alternatives, repeated in Figure 4.2, are suggested in the Report of Rome (Meadows et al. 1972). In $A$, population growth decreases linearly; in $C$, the negative effect is exponential, and in $B$ mortality increases as pollution bypasses a threshold level. Let us concentrate on case $B$ because the existence of a threshold is often discussed (see Meadows et al. 1972).

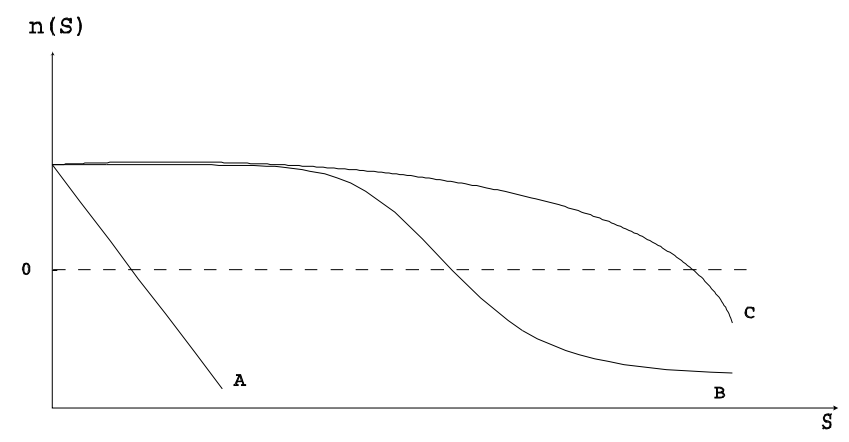

Figure 4.2: Possible functional formulas for the positive check. Meadows et al. 1972.

One of the simplest algebraic expressions that produces $B$ is

$$
n(S)=\beta-\frac{\alpha}{1+(\mu S)^{-\gamma}}
$$

in which $\beta$ gives the population growth for $S=0, \beta-\alpha$ gives the lowest population growth reached, $\mu>0$ multiplies the effect of $S$ such that large values of $\mu$ lead to negative population growth at low concentrations. Finally, 
$\gamma>0$ gives the curvature of the function with high values referring to the curved shape and severity of the crisis after the threshold. Further, let us assume that the abatement function takes the logistic formula

$$
\delta(S)=r S\left(1-\frac{S}{\tilde{S}}\right)
$$

in which $r>0$ is the intrinsic rate of decay.

Let conventional values $\theta=3$ and $\rho=0.04$ describe the preferences (see Barro and Sala-i-Martin 1995). Further, let $r=0.15$ and $\tilde{S}=1000$ describe the abatement function. Let the demographic parameters be $\beta=0.005$, referring to $0.5 \%$ population growth rate for $S=0$, and $\alpha=0.01$, indicating that the lowest population growth is $-0.5 \%$, a value that seems to be rather modest rather. Further, let $\mu=0.002$ and $\gamma=6$. These parameters imply that $\hat{S}=$ $\frac{1}{2} \tilde{S}=500$, i.e., population starts to decrease as the concentration reaches half of the carrying capacity. The model has a steady state at $S^{*}=683$ and the steady state population growth rate is $n\left(S^{*}\right)=-0.0037$, so that in the steady state the population halves every 187 years, whereas per capita consumption doubles in the same time. The depicted off-steady-state path for population shows that the critical value $\hat{S}=500$ is reached in only about 20 years because the positive population growth increases the pace of pollution initially. From this on, population decreases, pollution accumulates much more slowly and per capita consumption (consumption) starts to increase after some 50 years. After some 250 years the population is only $40 \%$ of its original size. 

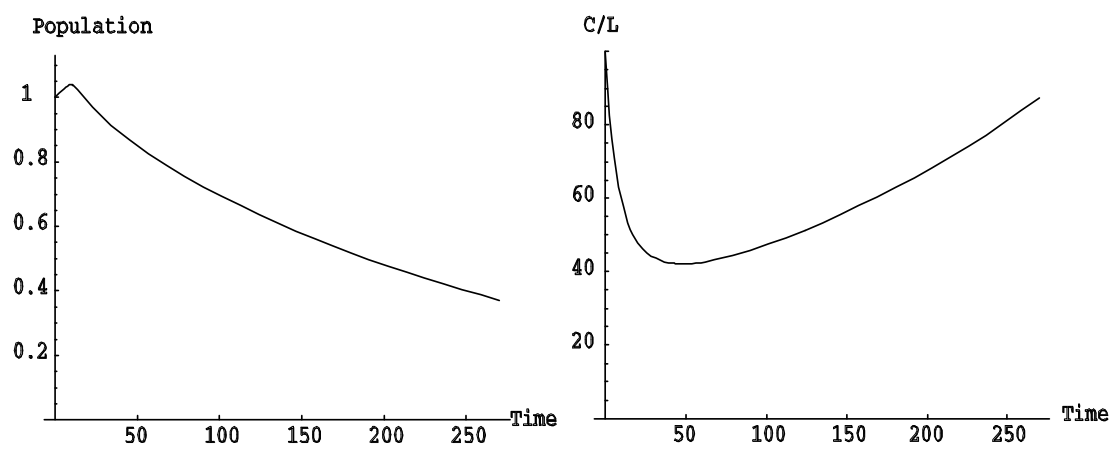

Figure 4.3: The parametric time paths for population and per capita consumption in the $n\left(S^{*}\right)=-0.0037$ case.

\section{Technical progress}

The optimists argue that technical progress will guarantee sustainability (Neumayer 1999). To see if this optimism is supported by the model, let $A(t)$ be the available technology at time $t$ and assume that technical progress is exogenously running at rate $x$ so that $A(t)=e^{x t}$ for $A(0)=1$. Further, let technical progress be consumption augmenting in the sense that at every instant of time $t$, we have $C=e^{x t} E$; for given emissions it is possible to consume more than before (Krautkraermer 1985). Per capita consumption then becomes

$$
C / L=e^{x t} E / L
$$

Per capita consumption $C / L$ grows at rate $\gamma_{C / L}=\dot{E} / E+x-n$. In a steady state, $\dot{E} / E=0$, so that $\gamma_{C / L}$ is positive if $x>n\left(S^{*}\right)$. It is thus possible to have growing per capita consumption and growing population together in the 
presence of technical progress.

However, positive population growth is by no means warranted. To see why, apply (5.1) to (3.4)-(3.9) to derive

$$
\frac{\dot{E}}{E}=0 \Leftrightarrow E=\frac{\theta-1}{\theta}\left\{\delta+\frac{\rho-n}{n^{\prime}}\left[\delta^{\prime}+(\theta-1) x+(\rho-\theta n)\right]\right\} .
$$

The derivative of (5.2) in terms of $x$ is:

$$
\left.\frac{\partial E}{\partial x}\right|_{\dot{E}=0}=\frac{(\theta-1)^{2}(\rho-n)}{\theta n^{\prime}}<0 .
$$

Therefore, the $\dot{E}=0$-line shifts down as the pace of technical progress increases (Figure 5.1).

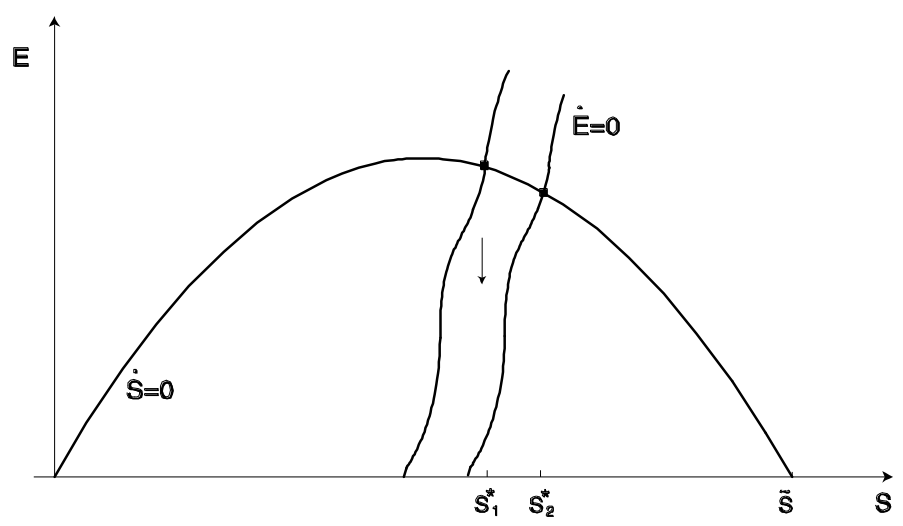

Figure 5.1: Technical progress shifts the $\dot{E}=0$-line down and increases the steady state pollution $S^{*}$.

To comprehend, note that in the equilibrium, the negative utility effect of a marginal emission through an increase in $S$ and a decrease in population 
growth, and its positive utility effect through an increase in consumption are equal and further emissions are rejected. Technical progress increases the positive consumption effect and larger emission are accepted. Given the biologically determined $\hat{S}$, it is then more likely that $\hat{S}<S^{*}$ and $n\left(S^{*}\right)$ is negative. Therefore, contrary to conventional wisdom, we find that technical progress does not necessarily save us because it makes extra consumption and emission pay.

To stipulate $\gamma_{C / L}=\dot{E} / E+x-n$ during the transitional period, write

$$
\frac{\dot{E}}{E}=\frac{n^{\prime}}{(\rho-n)(\theta-1)}\left\{\dot{S}+\frac{1}{\theta}\left[\delta-\frac{(\rho-n)(\theta-1)}{n^{\prime}}\left[\delta^{\prime}+\rho-\theta n+(\theta-1) x\right]\right]\right\},
$$

where the leftmost element is the positive difference of the $\dot{S}=0$ and $E=$ 0 -lines indicating $\dot{E} / E<0$ along the north-western saddle path. Further, as earlier, we have $\lim _{S \rightarrow S^{*}} \dot{E} / E=0$. Therefore, the sign of $\lim _{S \rightarrow S^{*}} \gamma_{C / L}$ depends on the sign of $x-n\left(S^{*}\right)$. In particular, for $n\left(S^{*}\right)<0$ we have $x-n\left(S^{*}\right)>0$ for all $x$ and $\lim _{S \rightarrow S^{*}} \gamma_{C / L}=\dot{E} / E+x-n>0$ implying that per capita consumption increases as the economy approaches the steady state $n\left(S^{*}\right)<0 .{ }^{8}$ The following proposition summarizes the results:

Proposition 1 If the optimal population growth in the steady state is negative, then per capita consumption increases as the economy approaches the steady state. A high rate of technical progress increases the probability for negative steady state population growth.

\footnotetext{
${ }^{8}$ The slope of the entire time path for $\gamma_{C / L}$ depends on $\lim _{S \rightarrow 0} \gamma_{C / L}$. This and the cases $n\left(S^{*}\right)>0$ and $n\left(S^{*}\right)=0$ are not considered for shortness.
} 


\section{Discussion}

The long run consumer optimization model with endogenous pollution and endogenous population implies that utility maximization may take place at the cost of human lives and may ultimately lead mankind to a self-imposed extinction. Solow has suggested that "The theory of optimal growth ... is thoroughly utilitarian in conception. It is also utilitarian in the narrow sense that social welfare is (usually) defined as the sum of the utilities of different individuals or generations" (Solow 1974). In the case of endogenous pollution and endogenous population, this utilitarianism may take an extreme expression: a path that ultimately leads to extinction may still be optimal. Naturally, a different result would have been derived if positive population were posed as an a priori constraint on optimization. However, an emerging empirical evidence suggests that there already is an increase in mortality because of environmental reasons. Therefore, as a description of the current situation, the utilitarian approach may not be so distorted after all.

An article on sustainable growth is, more or less, a wake-up call. Broadly speaking, one wants to predict what may happen if the currently shown disturbing behavior continues and if environmental concerns are not taken seriously. Therefore, it is important to give the right signal. With population exogenous, the warning signal for failing sustainability is that per capita consumption is decreasing but with population endogenous, increasing per capita consumption signals that the economy may be approaching a state in which population decreases forever. Naturally, this result of a theoretical model should be projected 
on to real world only with care. Currently, some people suffer and die but the vast majority consumes ever more. Therefore, if per capita consumption keeps increasing and if pollution-related mortality remains tolerable, the worrisome conclusion is that, in real world as well as in the model, the incentives for a change in economic behavior may not be sufficient.

\section{References}

BArbier, E. B. (2003): "The Role of Natural Resource in Economic Development," Australian Economic Papers, pp. 253-272.

Barro, R. J., and X. Sala-I-Martin (1995): Economic Growth. McGrawHill, New York.

Benveniste, L., And J. Scheinkman (1982): "Duality Theory for Dynamic Optimization Models of Economics: The Continuous Time Case," Journal of Economic Theory, 27, 1-19.

Brunekreef, B., and S. T. Holgate (2002): "Air Pollution and Health," The Lancet, 360, 1233-1242.

Chay, K. Y., and M. Greenstone (2003): "Air Quality, Infant Mortality, and the Clean Air Act of 1970," Working paper, MIT.

Currie, J., And M. Neidell (2004): "Air Pollution and Infant Health: What Can We Learn From California's Recent Experience?," Working Paper 10251, NBER. 
Dasgupta, P., and G. Heal (1974): "The Optimal Depletion of Exhaustible Resources," Review of Economic Studies, 41, 3-28.

Evans, M. F., And K. V. Smith (2005): "Do New Health Conditions Support Mortality-Air Pollution Effects," Journal of Environmental Economics and Management, 50, 496-518.

Foster, B. A. (1973): "Optimal Consumption Planning in a Polluted Environment," Economic Record, pp. 534-545.

Hall, R. E. (1988): "Intertemporal Substitution in Consumption," Journal of Political Economy, 96(2), 339-357.

IPCC (2001): Climate Change 2001, Ipacts, Adaptation, and Vulnerability. Intergovernmental Panel on Climate Change, The Third Assessment, Cambridge University Press, Cambridge, US.

Keeler, E., M. Spence, and R. Zechhauser (1971): "The Optimal Control of Pollution," Journal of Economic Theory, 4, 19-34.

Krautkraermer, J. A. (1985): "Optimal Growth, Resource Amenities and the Preservation of Natural Environments," Review of Economic Studies, $52(1), 153-170$.

Krutilla, J. V. (1967): “Conservation Reconsidered," American Economic Review, 57(4), 777-786.

LogAn, W. P. D. (1953): "Mortality in the London Fog Incident, 1952," Lancet, pp. 336-338. 
Malthus, T. R. (1914): An Essay on the Principle of Population. J. M. Dent and Sons LTD, 1973 Reprint, London.

Meadows, D., D. Meadows, E. Zahn, and P. Milling (1972): The Limits to Growth. Universe Books, New York.

Nemery, B., P. H. M. Hoet, and A. Nemmar (2001): "The Meuse Valley Fog 1930: An Air Pollution Disaster," Lancet, 357, 704-708.

Neumayer, E. (1999): Weak versus Strong Sustainability. Edward Elgar, Cheltenham, UK.

Pezzey, J. C. (1992): "Sustainable Development Concepts," Working Paper 11425, World Bank, Environment Department.

Pezzey, J. C., and C. A. Withagen (1998): "The Rise, Fall and Sustainability of Capital-Resource Economies," Scandinavian Journal of Economics, $100(2), 513-527$.

Plourde, C. (1972): "A Model of Waste Accumulation and Disposal," Canadian Journal of Economics, 5(1), 119-125.

Pope III, C. A., R. T. Burnett, M. J. Thun, E. E. Calle, D. Krewski, K. Ito, and G. D. Thurston (2002): "Lung Cancer, Cardiopulmonary Mortality, and Long-term Exposure to Fine Particulate Air Pollution," Journal of American Medical Association, 287(9), 1132-1141. 
Samet, J. M., F. Dominici, F. C. Curriero, I. Coursac, and S. L. Zeger (2000): "Fine Particulate Air Pollution and Mortality 20 U.S. Cities, 19871994," The New England Journal of Medicine, 343(22), 1742-1749.

Smulders, S., and R. Gradus (1996): "Pollution Abatement and Long-Term Growth," European Journal of Political Economy, 12, 505-532.

Solow, R. M. (1974): "Intergenerational Equity and Exhaustible Resources," Review of Economic Studies, 41, 29-45.

Stiglitz, J. (1974): "Growth with Exhaustible Natural Resources: Efficient and Optimal Growth Paths," Review of Economic Studies, 41, 123-137.

Tahvonen, O., and S. Salo (1996): "Nonconvexities in Optimal Pollution Accumulation," Journal of Enviromental Economics and Management, 31, $160-177$.

Tanser, F. C., B. Sharp, and D. Le Sueur (2003): "Potential Effect of Climate Change on Malaria Transmission in Africa," The Lancet, 362, 17921798.

United Nations (2005): World Population Prospects. The 2004 Revision. New York.

Uzawa, H. (1968): "Time Preference, the Consumption Function, and Optimum Asset Holdings," in (Wolfe 1968), chap. 21.

WCED (1987): Our Common Future. World Comission on Economic Development WCED, Oxford University Press, New York. 
WHO (2003): Climate Change and Human Health, Risks and Responses. New York.

WHO (2004a): The Effects of Air Pollution on Children's Health and Development: A Review of the Evidence. WHO Regional Office for Europe, Copenhagen.

WHO (2004b): Meta-analysis of Time-Series Studies and Panel Studies of Particulate Matter (PM) and Ozone (O3). WHO Regional Office for Europe, Copenhagen.

Wolfe, J. (ed.) (1968): Value, Capital, and Growth. Aldine, Chicago. 


\section{A Appendix: Local Stability of the steady states}

Let us write $\dot{S}=\varphi(S, E)$ and $\dot{E}=\phi(S, E)$. The Jacobian of the model is

$$
J=\left[\begin{array}{ll}
\varphi_{S} & \varphi_{E} \\
\phi_{S} & \phi_{E}
\end{array}\right] .
$$

As evaluated around the steady state, its elements become

$$
\begin{aligned}
\varphi_{S}= & -\delta^{\prime}, \\
\varphi_{E}= & 1, \\
\phi_{S}= & \frac{E}{\theta}\left\{\frac{-n^{\prime \prime}(\rho-n)-\left(n^{\prime}\right)^{\wedge}}{(\rho-n)^{2}}\left[\frac{\theta E}{1-\theta}+\delta\right]-\frac{n^{\prime}}{\rho-n}\left[\delta^{\prime}\right]-\left[\delta^{\prime \prime}-\theta n^{\prime}\right]\right\}, \\
\phi_{E}= & \frac{1}{\theta}\left\{\frac{n^{\prime}}{\rho-n}\left[\frac{\theta E}{\theta-1}+\delta\right]-\left(\delta^{\prime}+\rho-\theta n\right)\right\} \\
& +\frac{E}{\theta}\left\{\frac{n^{\prime}}{\rho-n} \frac{\theta}{\theta-1}\right\} \\
= & \frac{n^{\prime} E}{(\rho-n)(\theta-1)},
\end{aligned}
$$

in which the last row is derived by using (3.12) and (3.11b). Because $\phi_{S}$ contains the undefined second derivative of $n(S)$, we write

$$
\begin{aligned}
D E T J & =\varphi_{S} \cdot \phi_{E}-\phi_{S} \cdot \varphi_{E} \\
& =\left[\left(-\frac{\varphi_{S}}{\varphi_{E}}\right)-\left(-\frac{\phi_{S}}{\phi_{E}}\right)\right]\left(-\varphi_{E}\right) \cdot \phi_{E} .
\end{aligned}
$$

The expression $\left(-\varphi_{E}\right) \cdot \phi_{E}=-\frac{n^{\prime} E}{(\rho-n)(\theta-1)}$ is positive. The expression in the square brackets is the difference in the slopes of the phase lines $\dot{S}=0$ and 
$\dot{E}=0$. In the steady state, the $\dot{E}=0$-line hits the $\dot{S}=0$-line from below and this expression is negative, implying $D E T J<0$. Therefore, the steady state is a saddle. Because $\varphi_{E}>0$, we have $\dot{S}>0(\dot{S}<0)$ above (below) the $\dot{S}=0$-line. Because $\phi_{E}<0$, we have $\dot{E}>0(\dot{E}<0)$ below (above) the $\dot{E}=0$-line as is depicted in Figure 3.1. 